\title{
Fuzzy Logic Application of Steady-state Harmonic Distortion Limits for the Time-varying Harmonics
}

\author{
J.H. Han, G. Jang \\ School of Electrical Engineering \\ Korea University \\ Campus of Science, Seoul 136-701 (Korea) \\ Phone/Fax number:+82 23290 4766/3692, e-mail: hanpro_@korea.ac.kr, gjang@korea.ac.kr
}

\begin{abstract}
Harmonic distortion limits recommended by most international standards are designed for the steady-state harmonics, which are typically calculated for planning purpose. A problem can occur, however, when the limits are applied to actual time-varying harmonics. This paper proposes a new fuzzy logic application to apply modified steady-state harmonic limits using total demand distortion, which is recommended in the IEEE standard 519-1992, to the actual measured harmonic distortions which varies with time. Fuzzy logic application can provide simple and easy implementation and reflect experts' knowledge. The result of fuzzy logic application indicates how close the current waveform is to a pure sinusoidal wave shape and also reveals whether this current harmonic distortion is within or outside the allowable limits. It is expected that this application can contribute for future developments of harmonics monitoring, evaluation, and mitigation strategies in the actual operating status of power system.
\end{abstract}

\section{Key words}

Fuzzy inference system (FIS), fuzzy logic, harmonics, power quality (PQ), time-varying signals

\section{Introduction}

Both electric utilities and customers have experienced an increase of harmonic current and voltage level, due to the diverse diffusion of electric power devices in residential, commercial, and industrial loads. It becomes a great concern for both electric utilities and customers to maintain power quality (PQ). To provide a reference guide for them, most existing international standards recommend harmonic distortion limits for the steadystate harmonics. The most common harmonic index is total harmonic distortion (THD) which is defined as the root mean square (RMS) of the harmonics expressed as a percentage of the fundamental component. The THD can be weighted to indicate the stress on various system devices [1]. Similarly, a weighted factor can be used for applying a steady-state harmonic limit to time-varying harmonics. This will be explained in detail later. Current distortion levels can also be characterised by a THD value, but it can be misleading when the fundamental load current is low. A high THD value for input current may not be a matter of significant concern if the load is light, since the magnitude of the harmonic current is low even though its relative distortion to the fundamental frequency is high. To avoid such ambiguity, IEEE Standard 519-1992 defined a new factor called the total demand distortion (TDD) which is similar to THD except that the distortion is expressed as a percentage of some rated or maximum load current magnitude, rather than as a percentage of the fundamental current [1], [2]. Thus, as the size of a customer load decreases with respect to the size of a system, the percentage of harmonic current that the customer is allowed to inject into the utility system increases. Both THD and TDD limits are typically applied to the estimated harmonics during the planning stage.

Although the fundamental function of the harmonic indices is to indicate how far the analyzed signal deviates from the nominal sinusoidal waveform, TDD values alone are not enough to precisely describe the harmonic distortion level for certain situations in the power system [3]. TDD should be related to the ratio, short circuit level $\left(I_{s c} / I_{L}\right)$, of the short circuit current $\left(I_{s c}\right)$ available at the point of common coupling (PCC) to the maximum fundamental load current $\left(I_{L}\right)$ which is calculated as the average current of the maximum demand for the preceding 12 months (see Table I) [1]. For example, if we assume that both short circuit level of 35 and 85 have the same value of TDD of $10 \%$, the former current distortion will be outside the allowable limit but the latter current distortion will be within the limits.

Table I. - Current Distortion Limits for General Distribution Systems (120V through 69kV)

\begin{tabular}{|c|c|c|c|c|c|c|}
\hline \multicolumn{7}{|c|}{ Maximum harmonic current distortion in percent of $I_{L}$} \\
\hline \multicolumn{7}{|c|}{ Individual harmonic order (odd harmonics) } \\
\hline$I_{S C} / I_{L}$ & $<11$ & $11 \leq \mathrm{h}<17$ & $17 \leq \mathrm{h}<23$ & $23 \leq \mathrm{h}<35$ & $35 \leq \mathrm{h}$ & $\mathrm{TDD}$ \\
\hline$<20$ & 4.0 & 2.0 & 1.5 & 0.6 & 0.3 & 5.0 \\
\hline $20<50$ & 7.0 & 3.5 & 2.5 & 1.0 & 0.5 & 8.0 \\
\hline $50<100$ & 10.0 & 4.5 & 4.0 & 1.5 & 0.7 & 12.0 \\
\hline $100<1000$ & 12.0 & 5.5 & 5.0 & 2.0 & 1.0 & 15.0 \\
\hline$>1000$ & 15.0 & 7.0 & 6.0 & 2.5 & 1.4 & 20.0 \\
\hline
\end{tabular}

However, field measurements clearly indicate that the voltage and current harmonics are time-varying signals due to continual changes in system configuration and 
load conditions. According to IEC 61000-4-30 standard, a monitoring procedure for PQ can be simplified by Fig. 1 [4]. It is easily found that the evaluation results are dependent on the accuracy of the transducers and the analysis methods. In other words, there are some uncertainties in measuring a harmonic index as follows:

- Current transducers inaccuracies

- Measurement instrumentation errors

- Changes in power system operating conditions

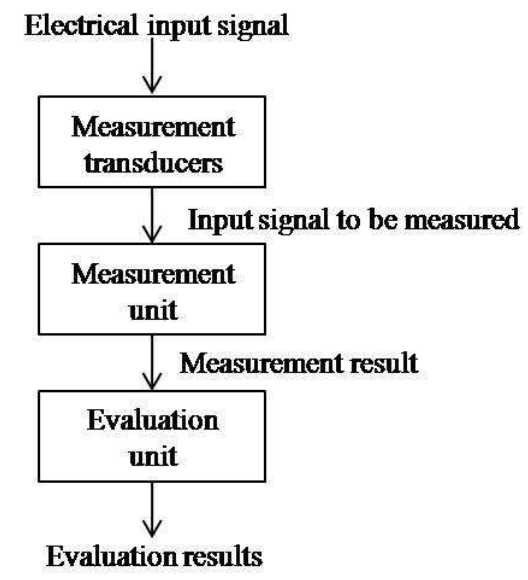

Fig. 1. PQ Monitoring Procedure

In several studies to define for the non-stationary signals, it is observed that the classical total harmonic distortion index limits the types of analysis approaches such as the Fourier transform, the Wavelet transform, or the other time-frequency representation [5]-[7]. Therefore, the accuracy of harmonic indices is highly dependent on the analysis transform-domain resolution. When a practical waveform is processed by windowed Fourier transform, time-varying harmonics can be detected. Discussion of this issue is however far outside the scope of this paper.

To make this simple, two sets of harmonic limits can be considered. One set is applied to the design (calculated) data and the other to the measured data. The design limits are recommended by the IEEE Standard 519-1992, which recognizes that they can be exceeded for periods of time without causing harm to equipment. However, the malfunction of electronic devices can be caused by such a short-duration burst of large harmonic distortions. To determine the short-duration harmonic limits for the measurement purpose, the design limits such as Table $\square$ was discussed in [10].

Table $\amalg$. - Short-duration Limits for Single Harmonic Burst

\begin{tabular}{|c|c|c|}
\hline $\begin{array}{c}\text { Maximum duration of } \\
\text { single harmonic } \\
\text { bursts }\left(\mathrm{T}_{\max }\right)\end{array}$ & $\begin{array}{c}\text { Maximum duration } \\
\text { of single harmonic } \\
\text { bursts }\left(\mathrm{T}_{\max } \text { in } \%\right)\end{array}$ & $\begin{array}{c}\text { Acceptable harmonic } \\
\text { distortion level }\end{array}$ \\
\hline $\mathrm{T}_{\max } \leq 15 \mathrm{sec}$. & $\mathrm{T}_{\max } \leq 0.02 \%$ & $3.0(\times$ design limits $)$ \\
\hline $15 \mathrm{sec} .<\mathrm{T}_{\max } \leq 30 \mathrm{~min}$. & $0.02 \%<\mathrm{T}_{\max } \leq 2 \%$ & 2.0 (xdesign limits $)$ \\
\hline $30 \min .<\mathrm{T}_{\max }$ & $2 \%<\mathrm{T}_{\max }$ & 1.0 (xdesign limits $)$ \\
\hline
\end{tabular}

This table takes into account the measurement resolution, or one measurement point for every 3 seconds, recommended by IEC 61000-4-30 and suggests that any single harmonic burst lasting more than 30 minutes over the 24 hour measurement period should be treated as steady-state harmonics. Although the limit values and duration are established by several factors such as engineering experiences, information described in various standards, and implementation issues, the actual limit values can always be adjusted as more experiences are gained.

The crucial problem is how to apply the steady-state harmonic limits to the actual time-varying harmonics concerning uncertainties. Although several PQ indices associated with harmonics are defined in some literatures, it is still difficult to observe the actual operation status of the power system in some practical applications with the indices [8], [9].

Therefore in this paper new fuzzy application which can handle uncertain, imprecise, and vague situations in measuring the TDD index will provide harmonic limits criteria taking into account time-varying harmonics. A fuzzy logic approach is proposed to calculate new fuzzy total demand distortion index (FTDDI) using the Mamdani-type fuzzy inference method which is the most commonly seen fuzzy methodology. This approach is simple to apply and useful to deal with uncertain problems. The flow of information of the basic fuzzy inference system (FIS) is illustrated schematically in Fig. 2.

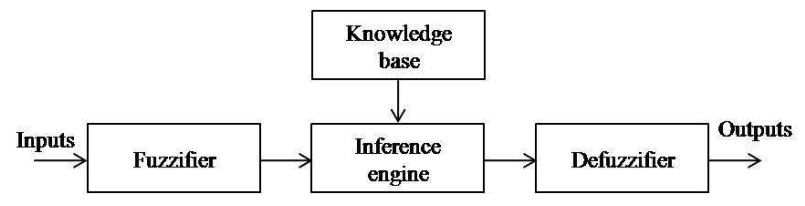

Fig. 2. Structure of Fuzzy Inference System

This paper is divided into six sections. The definition of TDD and fundamental of fuzzy inference system is reviewed in section $\Pi$ II and $I I$ respectively. Then the design of the fuzzy inference system is proposed in section IV. The simulation study results and analyses are presented in section $\mathrm{V}$ and finally conclusions are given at the end.

\section{Total Demand Distortion}

The IEEE Standard 519-1992 defined TDD as the total root mean square harmonic current distortion in percent of the maximum demand load current

$$
T D D=\sqrt{\frac{\sum_{h=2}^{\infty} I_{h}^{2}}{I_{L}^{2}}}
$$

where $I_{L}$ is the peak, or maximum, demand load current at the fundamental frequency component measured at the point of common coupling (PCC). This harmonic distortion limits which are listed in Table I provide the maximum allowable current distortion for a customer and should be used as system design values for the worst case for normal operation which is the conditions lasting longer than one hour. It should be also noticed that the limits may be exceeded by $50 \%$ for shorter periods [1]. 


\section{Fuzzy Inference System}

\section{A. Fuzzy Logic}

Fuzzy logic starts with the concept of a fuzzy set which is a set without a crisp, clearly defined boundary. A fuzzy set describes vague concept with the possibility of partial membership. The degree of input space belonging to a fuzzy set is denoted by a membership value between 0 and 1. A membership function (MF) associated with a given fuzzy set maps an input value to the appropriate membership value. For example, if $\mathrm{X}$ is the universe of discourse, or input space, a classical set might be expressed as

$$
A=\{x \mid x>6\}
$$

If $\mathrm{X}$ is the universe of discourse and its elements are represented by $\mathrm{x}$, a fuzzy set $\mathrm{B}$ in $\mathrm{X}$ is defined as a set of ordered pairs:

$$
B=\left\{x, \mu_{B}(x) \mid x \in X\right\}
$$

Here, $\mu_{B}(x)$ is the MF of $\mathrm{x}$ in the fuzzy set $\mathrm{B}$.

After fuzzy sets and fuzzy operators such as AND, OR, and NOT are decided, an if-then rule is applied to formulate the conditional statements that comprise fuzzy logic. A general fuzzy if-then rule can be represented as

\section{If (antecedent) then (consequent)}

The antecedent of a rule can also have multiple parts, and a fuzzy rule defines the linguistic connection between input and output.

\section{B. Fuzzy Inference System}

Fuzzy inference is the formulating process to map a given input to an output using fuzzy logic. The Mamdanitype fuzzy inference method is the most commonly used and has five parts of the fuzzy inference process: fuzzification of the input variables, application of the fuzzy operator in the antecedent, implication from the antecedent to the consequent, aggregation of the consequents across the rules, and defuzzification.

1) Fuzzify Inputs: This step is to take inputs and determine the fuzzy degree of membership in qualifying linguistic set.

2) Apply Fuzzy Operation: In this step, if the antecedent of a given rule has more than one part, a fuzzy operator has to be applied to get one number that represents the result of antecedent. Then the output will be a single truth value. There are the most common fuzzy operators: AND or OR

3) Apply Implication Method: The input for an implication process is a single number given by the output value of the previous step, and the output is a fuzzy set. Implication is implemented for each rule and several operators can be used such as MIN (minimum) truncating the output fuzzy set or PROD (product) scaling the output fuzzy set.

4) Aggregate All Outputs: Aggregation is the process by which the fuzzy sets representing the outputs of each rule are combined into a single fuzzy set. An input of the aggregation process is the list of truncated or scaled output fuzzy sets returned by the implication process for each rule, and the output of the aggregation process is one fuzzy set for each output variable. Several methods can be applied in this step such as maximum or the sum of each rule's output set.

5) Defuzzify: This step provides an output with a single number from the aggregate fuzzy set. The most popular defuzzification method is the centroid calculation which returns the center of area under the graph.

\section{Proposed FIS Implementation}

The proposed fuzzy logic application is used to calculate FTDDI, which is an index to denote the polluted current waveform from the harmonic distortion and to decide whether the current harmonic distortion is within the allowable limits or not. Fig. 3 shows very compact process of the FIS to get FTDDI from linguistic variable fuzzification all the way through defuzzifification of the aggregate output. This process was designed by Fuzzy Logic Toolbox available in Matlab [11].

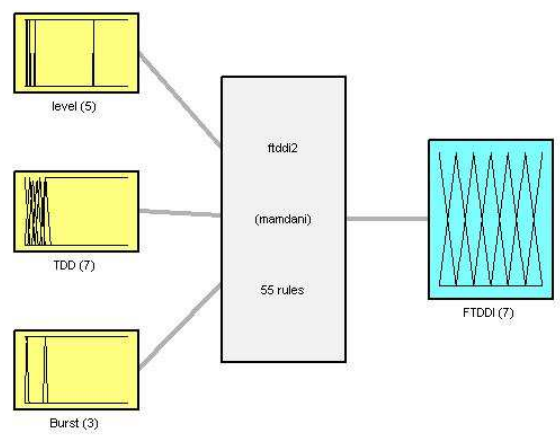

System ftddiz: 3 inputs, 1 outputs, 55 rules

Fig. 3. FIS Diagram for FTDDI

\section{A. Input Fuzzification}

The inputs of FIS are the short circuit level, the duration of single harmonic burst, and TDD. Trapezoidal membership function is suitable to represent the range of short circuit level because it has a part of identical value. Five linguistic variables are used to denote the short circuit level: low (L), slightly low (SL), medium (M), slightly high $(\mathrm{SH})$, and high $(\mathrm{H})$. Each linguistic variable is designed to cover one of the ranges of the short circuit levels listed in Table I. For example, the linguistic variable low (L) means under the short circuit level 50, while the linguistic variable slightly low (SL) is used to represent between the short circuit level 20 and 50 and so 
on. Similarly, trapezoidal membership function is applicable to represent the duration of single harmonic burst which is the second input of the FIS, since it is required to identify the weighted factor as listed in Table $\Pi$. Three linguistic variables are used: short (S), medium (M), and long (L). For example, short (S) means under the $0.02 \%$ of single harmonic burst duration over the whole measurement time, while long (L) is used to identify the maximum duration of single harmonic burst over 30 minutes.

On the other hand, triangular membership functions are used to represent both the third input TDD and the consequent of each rule. Seven linguistic variables are used to identify the limits of the TDD according to Table $\mathrm{I}$ in the same way as above inputs: very low (VL), low (L), somewhat low (SL), medium (M), somewhat high $(\mathrm{SH})$, high $(\mathrm{H})$, and very high $(\mathrm{VH})$.

The consequent of each rule also has seven linguistic variables such as low (L), moderately low (ML), somewhat low (SL), medium $(\mathrm{M})$, somewhat high $(\mathrm{SH})$, moderately high $(\mathrm{MH})$, and high $(\mathrm{H})$ like the preceding. These outputs are used to include the whole range of the FTDDI from 0 to 1 . All input and output membership functions are shown in Fig. 4.

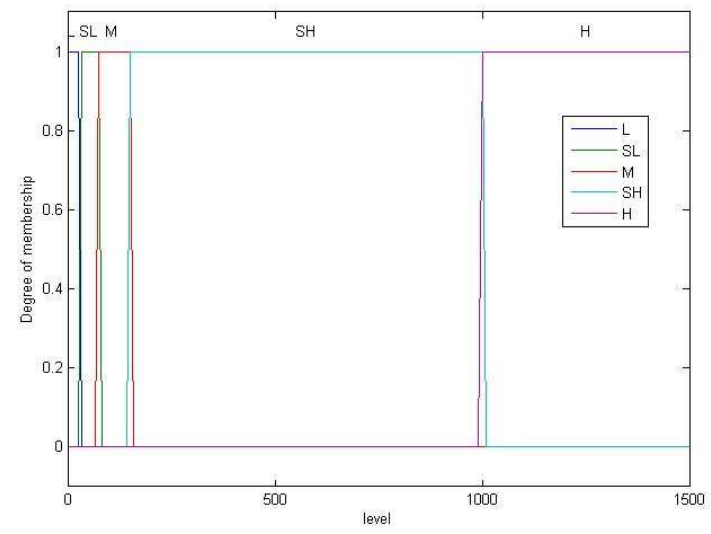

(a) Short Circuit Level

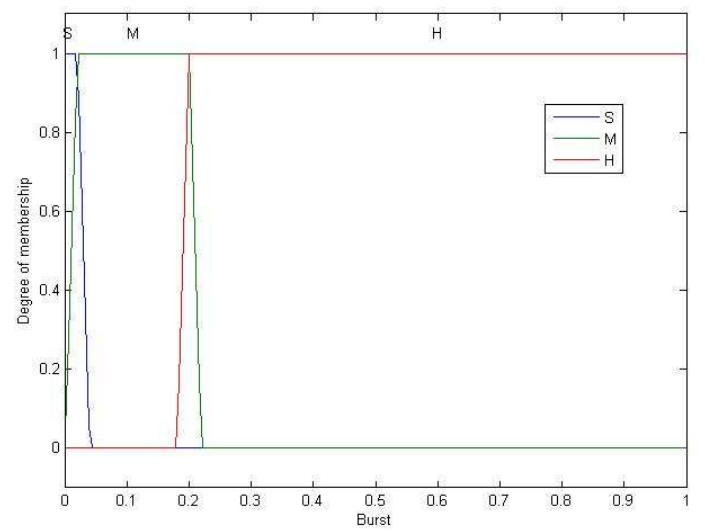

(b) Duration of Single Harmonic Burst

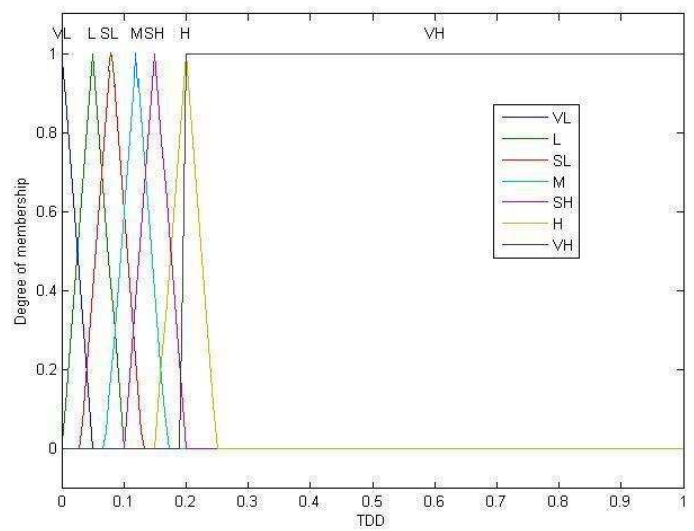

(c) Total Demand Distortion

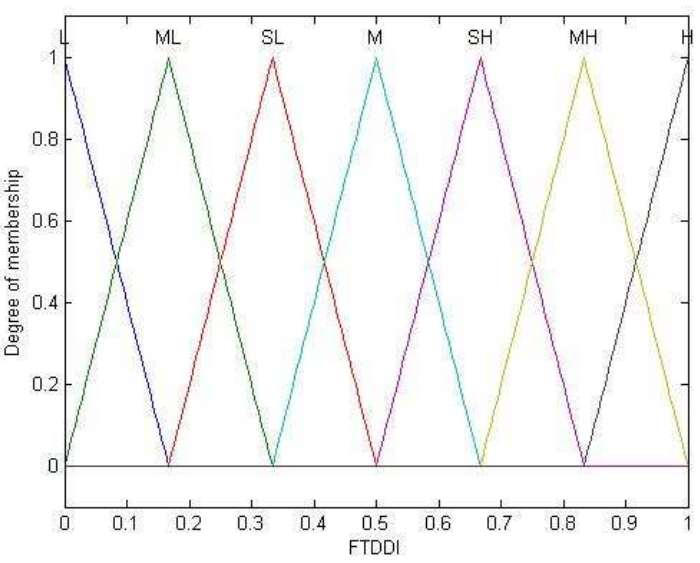

(d) FTDDI

Fig. 4. Input and Output Membership Function

\section{B. Fuzzy Rules}

There are 55 rules for the proposed FIS implementation as follows.

1) If (short circuit level is $L)$ and (TDD is VL) then (FTDDI is $H$ ).

2) If (short circuit level is L) and (TDD is L) then (FTDDI is $M H)$

3) If (short circuit level is L) and (duration of single harmonic burst is $S$ ) and (TDD is L) then (FTDDI is MH).

4) If (short circuit level is L) and (duration of single harmonic burst is $M)$ and (TDD is L) then (FTDDI is MH).

5) If (short circuit level is $L$ ) and (TDD is $S L$ ) then (FTDDI is $\mathrm{SH}$ ).

6) If (short circuit level is L) and (duration of single harmonic burst is $S$ ) and (TDD is $S L)$ then (FTDDI is SH).

7) If (short circuit level is L) and (duration of single harmonic burst is $M$ ) and (TDD is SL) then (FTDDI is SH).

8) $\sim 48)$

49) If (short circuit level is $H$ ) and (TDD is $S H$ ) then (FTDDI is $H$ ). 
50) If (short circuit level is $H$ ) and (TDD is $H$ ) then (FTDDI is MH).

51) If (short circuit level is $H$ ) and (duration of single harmonic burst is $S$ ) and (TDD is H) then (FTDDI is MH).

52) If (short circuit level is $H$ ) and (duration of single harmonic burst is $M$ ) and (TDD is $H$ ) then (FTDDI is MH).

53) If (short circuit level is $H$ ) and (TDD is VH) then (FTDDI is $S H$ ).

54) If (short circuit level is $H$ ) and (duration of single harmonic burst is $S$ ) and (TDD is VH) then (FTDDI is SH).

55) If (short circuit level is $H$ ) and (duration of single harmonic burst is $M$ ) and (TDD is VH) then (FTDDI is SMH).

The antecedent parts of each rule are designed by different combinations of the input linguistic variables using the 'and' operator, while the consequent part of each rule is decided to match the output linguistic variables and proper value for the FTDDI. For example, if short circuit level is low and TDD is very low, then the FTDDI should be a very high value to indicate that it is within the allowable limits. On the other hand, if the short circuit level is low and TDD is very high, then the FTDDI should be low to show proper level of harmonic distortion outside of limits. The critical parts of these rules are when the FTDDI is around threshold of limits because FTDDI is crucially influenced herein by how long the single harmonic bursts last. Both the consequent linguistic variable 'somewhat high' and 'moderately high' of output membership function have something to do with the threshold of limit a lot. Therefore, twenty rules which are related with the consequent linguistic variable 'somewhat high' and 'moderately high' of output membership function additionally consider the duration of single harmonic burst as an input variable. The weighted factor specified by Table $\Pi$ is individually applied to each rule of them.

\section{Fuzzy Operator}

The fuzzy operator minimum is used in the implication process, while the fuzzy operator maximum is used in aggregation part.

\section{Defuzzification}

The center of area (COA) method is used to deffuzify the aggregate fuzzy set. For given values of the short circuit level, TDD, and duration of single harmonic burst, the FIS calculates FTDDI.

\section{Applications and Results}

To test the validity, performance and feasibility of the proposed FTDDI, a utility distribution feeder example introduced by IEEE standard 519-1992 is applied in this paper. This distribution system feeder which has four users along the feeder is shown in Fig. 5.

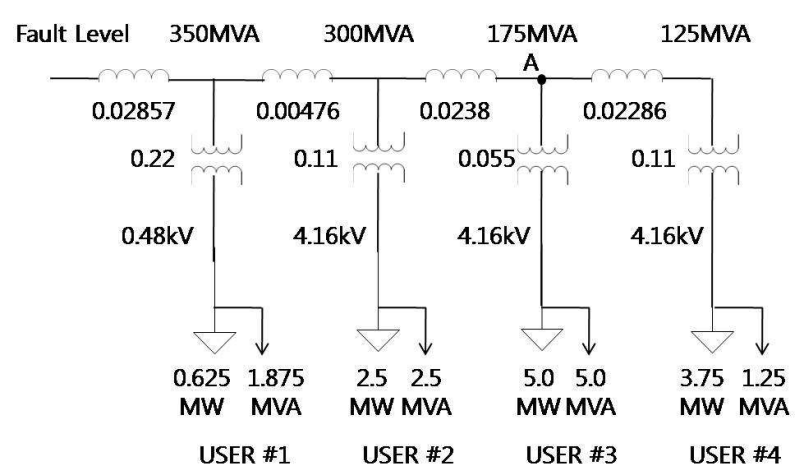

Fig. 5. Single-line Diagram of Distribution System Feeder

Each user has a different value of short circuit level, and the current distortion from each user is caused by static power converter load. As usual, the TDD is measured at the PCC for each user. Note that the values of TDD for both user \#1 and user \#4 are well within the IEEE standard 519-1992 limits. On the other hand, the values of TDD for both user \#2 and user \#3 are over the limits as specified by Table $\mathbb{\text { II. }}$.

Table III. - Harmonic Distortion without Filter

\begin{tabular}{|c|c|c|c|c|}
\hline & User \#1 & User \#2 & User \#3 & User \#4 \\
\hline $\mathrm{I}_{\mathrm{SC}} / \mathrm{I}_{\mathrm{L}}$ & 140 & 60 & 17.5 & 1405 \\
\hline $5^{\text {th }}$ & 4.80 & 9.67 & 9.59 & 14.4 \\
\hline $7^{\text {th }}$ & 3.33 & 6.65 & 6.60 & 9.90 \\
\hline $11^{\text {th }}$ & 1.83 & 3.67 & 3.66 & 5.50 \\
\hline $13^{\text {th }}$ & 1.42 & 2.87 & 2.85 & 4.28 \\
\hline $17^{\text {th }}$ & 0.87 & 1.76 & 1.75 & 2.63 \\
\hline $19^{\text {th }}$ & 0.67 & 1.36 & 1.35 & 2.03 \\
\hline $23^{\text {rd }}$ & 0.50 & 1.00 & 1.00 & 1.50 \\
\hline $25^{\text {th }}$ & 0.40 & 0.80 & 0.80 & 1.20 \\
\hline $29^{\text {th }}$ & 0.35 & 0.70 & 0.70 & 1.05 \\
\hline $31^{\text {st }}$ & 0.30 & 0.60 & 0.60 & 0.90 \\
\hline $35^{\text {th }}$ & 0.28 & 0.56 & 0.55 & 0.82 \\
\hline${ }^{2}$ & $6.42 \%$ & $13.0 \%$ & $12.8 \%$ & $19.3 \%$ \\
& $(15 \%)$ & $(12 \%)$ & $(5.0 \%)$ & $(20.0 \%)$ \\
\hline
\end{tabular}

* A percentage in parenthesis refers to the limit.

In such a case, one of the general solutions can be to place a harmonic filter near user \# 3 to mitigate the harmonic currents created by user \#3 and \#4. Around 4.1 Mvar of capacitors are designed to remove $5^{\text {th }}$ harmonic current and are furnished at point $\mathrm{A}$ in Fig. 5. There is, however, still no consideration for the time-varying characteristic of current distortion.

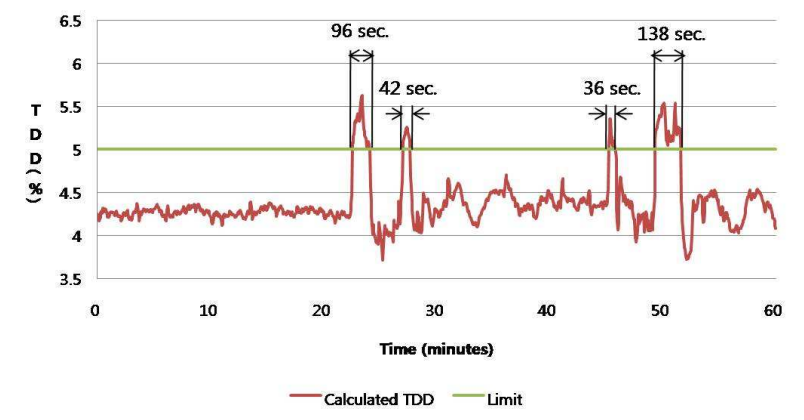

Fig. 6. Time-varying Harmonic Distortion 
On the surface, the conventional TDD is well within acceptable limits for every user on the distribution feeder with a filter as specified by Table IV. It seems a costeffective solution to mitigate current harmonic distortion in this distribution system feeder even though the timevarying characteristic of harmonic distortion is often displayed during one week at the PCC as shown in Fig. 6. However, existing TDD does not give any information of time-varying harmonic distortion because the TDD is defined as the steady-state concept of harmonics. Therefore, a new index is needed to quantify the severity of time-varying harmonic distortion for a real situation. The proposed FTDDI can serve this purpose, given that the TDD varies with time. To generate time-varying harmonics such as Fig. 6, a practical current waveform is processed by windowed Fourier transform. Note that a single data point is an average of many sampled cycles within 3 seconds. It is further assumed that the measurement period is 60 minutes which applies to any time of a day. In the view of time-varying TDD, the FTDDI is suitable for deciding whether the distortion contained in the current is within acceptable limit or not. The value of FTDDI for the user \#3 is 0.76 less than 0.78 . This result indicates that the harmonic distortion is outside the limits even though the value of TDD is within the IEEE standard 519-1992 limits.

Table IV. - Harmonic Distortion with Filter

\begin{tabular}{|c|c|c|c|c|}
\hline & User \#1 & User \#2 & User \#3 & User \#4 \\
\hline $\mathrm{I}_{\mathrm{SC}} / \mathrm{I}_{\mathrm{L}}$ & 140 & 60 & 17.5 & 1405 \\
\hline $5^{\text {th }}$ & 2.39 & 4.03 & 0 & 0 \\
\hline $7^{\text {th }}$ & 2.39 & 4.52 & 2.99 & 4.49 \\
\hline $11^{\text {th }}$ & 1.46 & 2.76 & 2.10 & 3.16 \\
\hline $13^{\text {th }}$ & 1.13 & 2.18 & 1.68 & 2.54 \\
\hline $17^{\text {th }}$ & 0.70 & 1.36 & 1.06 & 1.60 \\
\hline $19^{\text {th }}$ & 0.54 & 1.05 & 0.83 & 1.24 \\
\hline $23^{\text {rd }}$ & 0.40 & 0.78 & 0.62 & 0.93 \\
\hline $25^{\text {th }}$ & 0.33 & 0.63 & 0.50 & 0.75 \\
\hline $29^{\text {th }}$ & 0.28 & 0.55 & 0.44 & 0.66 \\
\hline $31^{\text {st }}$ & 0.24 & 0.47 & 0.37 & 0.66 \\
\hline $35^{\text {th }}$ & 0.22 & 0.44 & 0.34 & 0.51 \\
\hline \multirow{2}{*}{$\mathrm{TDD}$} & $4.00 \%$ & $7.35 \%$ & $4.37 \%$ & $6.57 \%$ \\
& $(15 \%)$ & $(12 \%)$ & $(5.0 \%)$ & $(20.0 \%)$ \\
\hline \multirow{2}{*}{ FTDDI } & 0.95 & 0.84 & 0.76 & 0.94 \\
& $(0.73)$ & $(0.64)$ & $(0.78)$ & $(0.75)$ \\
\hline
\end{tabular}

* A percentage in parenthesis refers to the limit.

Therefore the only TDD may give misleading answers about whether the time-varying harmonic current distortion is acceptable or not if we apply the steady-state harmonic limits to the actual time-varying harmonics without any modification. The key idea of the proposed FTDDI is to apply modified steady-state harmonic limits to the short-term duration characteristics of the measured harmonic distortions. This is due to the fact that the FTDDI considers the short-term single harmonic burst together with the short circuit level and TDD.

\section{Conclusion}

This paper identifies the need to develop a set of limits for measured or time-varying harmonics. The proposed fuzzy logic application to obtain FTDDI has the advantage of being simple to implement and contains knowledge base so there is no need for an expert's skill after the designing stage. As the results obtained from different cases in the distribution system feeder, the proposed FTDDI provides two important data which cannot be obtained from the TDD alone. It indicates how much current waveform is distorted by time-varying harmonics and whether the distortion is within allowable limits or not. The proposed FTDDI can be a very effective index to evaluate time-varying harmonic distortion and to limit time-varying harmonics for the purpose of measurement in the real world. With these concepts and procedures, the specific values can be applied to the actual field as more experiences are gained.

\section{Acknowledgement}

This work was supported by the National Research Foundation of Korea (NRF) grant funded by the Korea government (MEST) (No. 2009-0083187) and by KESRI (R-2007-2-055) which is funded by Ministry of Knowledge Economy.

\section{References}

[1] IEEE Recommended Practices and Requirements for Harmonic Control in Electric Power Systems, IEEE Std. 519-1992, 1993.

[2] J. Arrillaga, N. R. Watson, and S. Chen, Power System Quality Assessment, John Wiley \& Sons Ltd, West Sussex (2000), pp. 38.

[3] W. G. Morsi and M. E. EL-Hawary, "A new fuzzy-based total demand distortion factor for nonsinusoidal situations", IEEE Trans. on Power Delivery, Vol. 23, No. 2, Apr. 2008, pp. 1007-1014.

[4] Testing and measurement techniques - Power quality measurements methods, IEC Std. 61000-4-30, 2003.

[5] S. G. Jaramillo, G. T. Heydt, and E. O'neill-Carrillo, "Power quality indices for aperiodic voltages and currents", IEEE Trans. on Power Delivery, Vol. 15, No. 2, Apr. 2000, pp. $784-790$

[6] M. S. Kandil, S. A. Farghal, and A. Elmitwally, "Refined power quality indices", IEE Proc. Generation, Transmission, and Distribution, Vol. 148, No. 6, Nov. 2001, pp. 590-596.

[7] Y. J. Shin, E. J. Powers, M. Grady, and A. Arapostathis, "Power quality indices for transient disturbances", IEEE Trans. on Power Delivery, Vol. 21, No. 1, Jan. 2006, pp. 253-261.

[8] W. Xu, "Application of steady-state harmonic distortion limits to the time-varying measured harmonic distortions", Power Engineering Society Summer Meeting 2002, Vol. 2, pp. 955-957.

[9] Task Force on Probabilistic Aspects of Harmonics (Y. Baghzouz, Chair), "Time-varying harmonics: Part ICharacterizing measured data", IEEE Trans. on Power Delivery, Vol. 13, Jul. 1998, pp. 938-944.

[10] C. I. Chen and G. W. Chang, "Review and comments on applications of harmonic indices", Power \& Energy Society General Meeting 2009, pp.1-5.

[11] Fuzzy Logic Toolbox user's Guide, The MathWorks (1999). 